\title{
Update on Repetitive Transcranial Magnetic Stimulation in Obsessive-Compulsive Disorder: Different Targets
}

\author{
Rianne M. Blom • Martijn Figee • Nienke Vulink • \\ Damiaan Denys
}

Published online: 6 May 2011

(C) The Author(s) 2011. This article is published with open access at Springerlink.com

\begin{abstract}
Obsessive-compulsive disorder (OCD) is a chronic, disabling disorder. Ten percent of patients remain treatment refractory despite several treatments. For these severe, treatment-refractory patients, repetitive transcranial magnetic stimulation (rTMS) has been suggested as a treatment option. Since 1997, in published trials, a total of 110 OCD patients have been treated with rTMS. This review aims to provide an update on rTMS treatment in patients with OCD. First, the mechanism
\end{abstract}

R. M. Blom

Department of Psychiatry, Academic Medical Center,

University of Amsterdam,

PA.3-127, P.O. Box 75867, 1070 AW, Amsterdam,

The Netherlands

e-mail: r.m.blom@amc.uva.nl

\section{Figee}

Department of Psychiatry, Academic Medical Center, University of Amsterdam,

PA.1-156, P.O. Box 75867, 1070 AW, Amsterdam,

The Netherlands

e-mail: m.figee@amc.uva.nl

\section{N. Vulink}

Department of Psychiatry, Academic Medical Center, University of Amsterdam,

PA.1-154, P.O. Box 75867, 1070 AW, Amsterdam,

The Netherlands

e-mail: n.c.vulink@amc.uva.nl

\section{Denys $(\bowtie)$}

Department of Psychiatry, Academic Medical Center, University of Amsterdam,

PA.2-179, P.O. Box 75867, 1070 AW, Amsterdam,

The Netherlands

e-mail: d.denys@amc.uva.nl

D. Denys

The Netherlands Institute for Neuroscience, an institute of the Royal Netherlands Academy of Arts and Sciences,

Amsterdam, The Netherlands of action is discussed, followed by the efficacy and side effects of rTMS at various brain targets, and finally implications for the future. Due to the lack of studies with comparable stimulation or treatment parameters and with reliable designs, it is difficult to draw clear conclusions. In general, rTMS appears to be effective in open-label studies; however, this has not yet been replicated in randomized, sham-controlled trials.

Keywords Repetitive transcranial magnetic stimulation . rTMS · Treatment refractory - Obsessive-compulsive disorder - Targets

\section{Introduction}

Obsessive-compulsive disorder (OCD) is a highly disablitating psychiatric disorder characterized by obsessions and compulsions. Obsessions are egodystonic, unwanted thoughts, images, or impulses that repeatedly enter one's mind. Compulsions are repetitive, time-consuming behaviors or mental acts often performed to neutralize the anxiety provoked by obsessions [1]. The prevalence of OCD in the general population is estimated at $1 \%$ to $3 \%$, and the disorder is associated with impaired functioning and decreased quality of life $[2,3]$. The general treatment of OCD is a serotonin reuptake inhibitor at an adequate dose, cognitive-behavioral therapy, or a combination of the two. However, up to $40 \%$ of patients fail to respond satisfactorily to these generally adequate treatment options, and $10 \%$ cannot be helped at all $[1,4]$.

With use of repetitive transcranial magnetic stimulation (rTMS), it has become possible to modulate local neural activity by inducing a depolarizing magnetic field pulse [5]. Because OCD may be related to increased neural activity in prefrontal subcortical circuits [6], the inhibitory effect of rTMS was hypothesized to be beneficial in OCD treatment. 
In 1997, Greenberg et al. [7] introduced rTMS as a new treatment approach for OCD. Earlier, rTMS had been shown to have a positive effect on mood disorders with stimulation of the prefrontal cortex [8]. Greenberg et al. [7] hypothesized that inhibition of the prefrontal activity with rTMS might reduce obsessive-compulsive symptoms. They applied rTMS ( $80 \%$ motor threshold, $20 \mathrm{~Hz}$ for $2 \mathrm{~s} / \mathrm{min}$ ) for $20 \mathrm{~min}$ to 12 patients with OCD and found significantly decreased compulsive urges for $8 \mathrm{~h}$ after stimulation. Since then, rTMS has been investigated in OCD, targeting several brain areas within the corticostriatal network. In this article, the mechanism of action is discussed first, then the efficacy and side effects of rTMS at various brain targets, and finally implications for the future.

\section{Mechanism of Action}

In the early-1980s, the transcranial magnetic stimulation (TMS) device was developed by Barker and colleagues [5]. The device stimulates the human cortex directly using a contactless and noninvasive method. It uses a strong pulse of electrical current that is sent through a coil to induce a magnetic field pulse in the area under the coil. This pulse has the capacity to depolarize superficial local neurons [5]. To create a longer lasting effect of the depolarized neurons, application of rTMS is needed. The magnitude and direction of rTMS-induced neuronal modulation depend on extrinsic factors such as motor threshold, frequency, and total number of stimuli, and intrinsic factors such as the functional state of the cortex [9]. For example, it appears that low-frequency rTMS $(0-5 \mathrm{~Hz})$ results in decreased neural excitability and regional cerebral blood flow, as opposed to high-frequency rTMS (5-20 Hz), which increases both [10].

Because knowledge of involvement of specific brain circuits in OCD is advancing, rTMS has been applied to several brain targets (Table 1). The rationale for the first rTMS studies in OCD was based on functional neuroimaging studies of OCD that demonstrated abnormalities in the orbitofrontal subcortical circuits, especially in the orbital frontal gyri and medial caudate nuclei [11]. This circuitry may be manipulated with rTMS by 1) stimulation of the dorsolateral prefrontal cortex (DLPFC) [12], 2) inhibition of the orbitofrontal cortex (OFC) directly [13], or 3) inhibition of the supplementary motor area (SMA). The SMA was chosen as a useful target for rTMS because it has extensive connections with regions implicated in cognitive processes and motor control $[14,15]$.

\section{Efficacy of Repetitive Transcranial Magnetic Stimulation in Obsessive-Compulsive Disorder}

A total of 110 OCD patients in 10 studies have been treated with rTMS, targeting the DLPFC, the OFC, or the SMA.
Four studies investigated the efficacy of rTMS in OCD in a double-blind, randomized, sham-controlled design $[12,16$, 18•, 19]; three studies in a sham-controlled design, although not double-blind $[13,17 \cdot, 20]$; and three case studies in an open fashion $[7,14,21]$. The characteristics of each study are summarized in Table 1 .

\section{Dorsolateral Prefrontal Cortex}

The DLPFC has been the most investigated target for rTMS in OCD. In 1997, Greenberg et al. [7] treated 12 OCD patients with rTMS to the right DLPFC, the left DLPFC, and lastly the midoccipital cortex as a control condition [7]. Eight of 12 patients were stable on serotonin reuptake inhibitor treatment. rTMS was randomly applied to these targets in an open fashion on separate days, at $80 \%$ threshold, $20 \mathrm{~Hz}$ for $2 \mathrm{~s} / \mathrm{min}$ for $20 \mathrm{~min}$. Compulsions, as measured by the Yale-Brown Obsessive Compulsive Scale (Y-BOCS), decreased significantly with $34.8 \%$ immediately after right DLPFC stimulation $(P<0.01)$ and remained significant $8 \mathrm{~h}$ afterward $(P<0.02)$, whereas obsessions did not decrease significantly. Depressive symptoms decreased significantly as well, although the effect did not last longer than $8 \mathrm{~h}$. Compulsions decreased instantly with 26.8\% $(P<0.03)$ following left DLPFC stimulation, but similar to depressive symptoms, they returned after $8 \mathrm{~h}$. Midoccipital stimulation increased compulsions, as measured by the Y-BOCS (nonsignificantly) $(P=0.07)$.

In 2001, Sachdev et al. [21] tried to replicate this study in 12 patients with treatment-resistant OCD. Right $(n=6)$ and left $(n=6)$ DLPFC stimulation was applied in an open fashion at $10 \mathrm{~Hz}, 100 \%$ motor threshold for 10 sessions of $2.5 \mathrm{~min}$. At 4 weeks of follow-up, rTMS led to a mean decrease on the Y-BOCS of 57\% for right DLPFC and $27 \%$ for left DLPFC. All 12 individuals were analyzed together, as there were no differences on any of the parameters measured, and showed a significant decrease of $42 \%$ on the Y-BOCS at 1-month follow-up $(P=0.003)$. However, after corrections for depression scores on the MontgomeryAsberg Depression Rating Scale, the significance disappeared $(P=0.06)$. In the same year, the first randomized, sham-controlled, double-blind rTMS OCD trial was completed. Alonso et al. [16] randomly assigned 18 patients with OCD to real rTMS $(n=10)$ or sham rTMS $(n=8)$ at the right DLPFC. The rTMS lasted $20 \mathrm{~min}$ at $1 \mathrm{~Hz}$ for both conditions, but the motor threshold was $110 \%$ for real rTMS and $20 \%$ for sham rTMS. This study failed to find significant improvement on the Y-BOCS or Hamilton Depression Rating Scale (HAM-D) after 18 sessions.

In 2006, this randomized, sham-controlled, double-blind design was repeated stimulating the left instead of the right DLPFC in 30 treatment-resistant OCD patients [19]. Patients were given 10 daily sessions of sham or real 


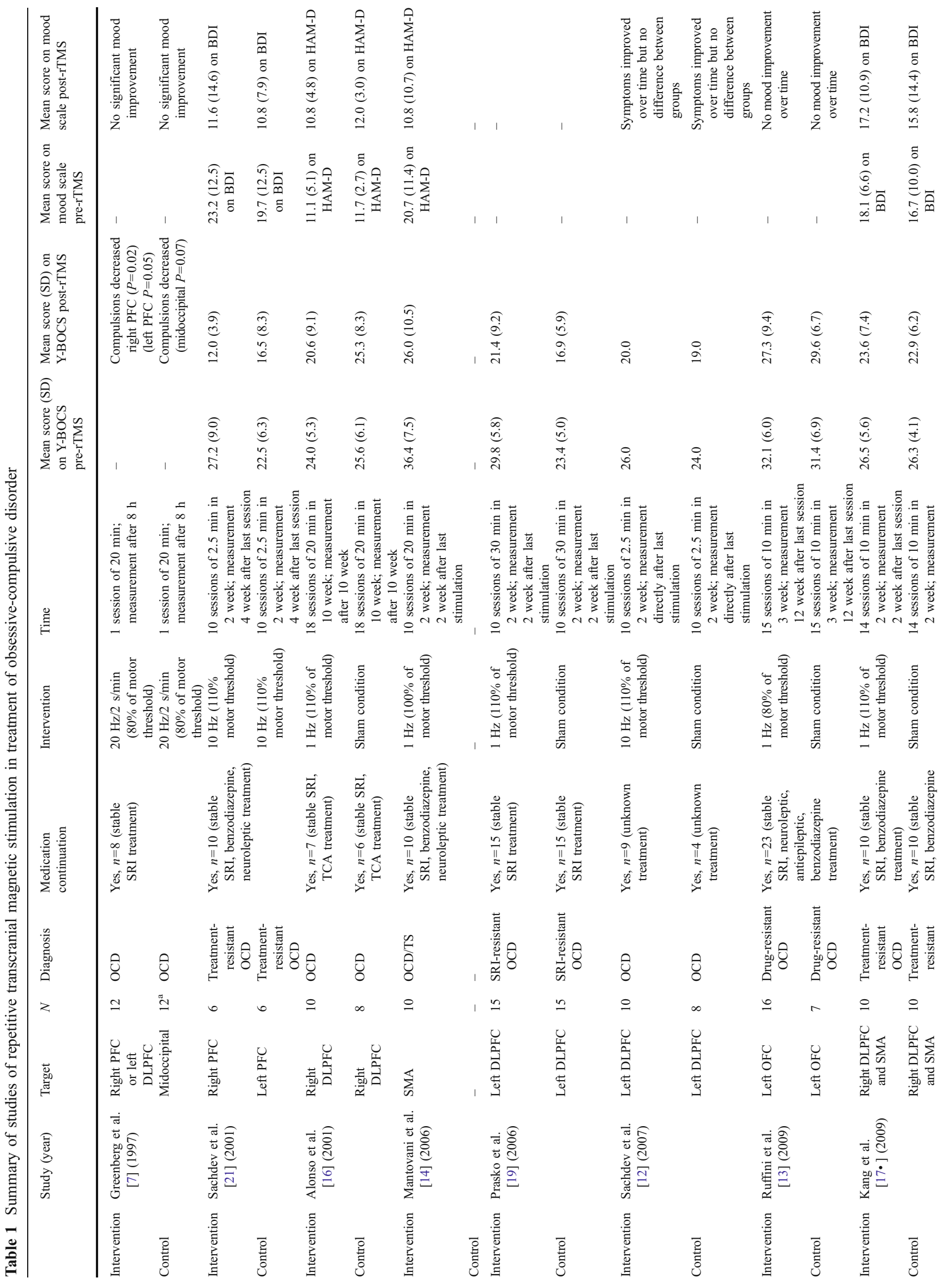




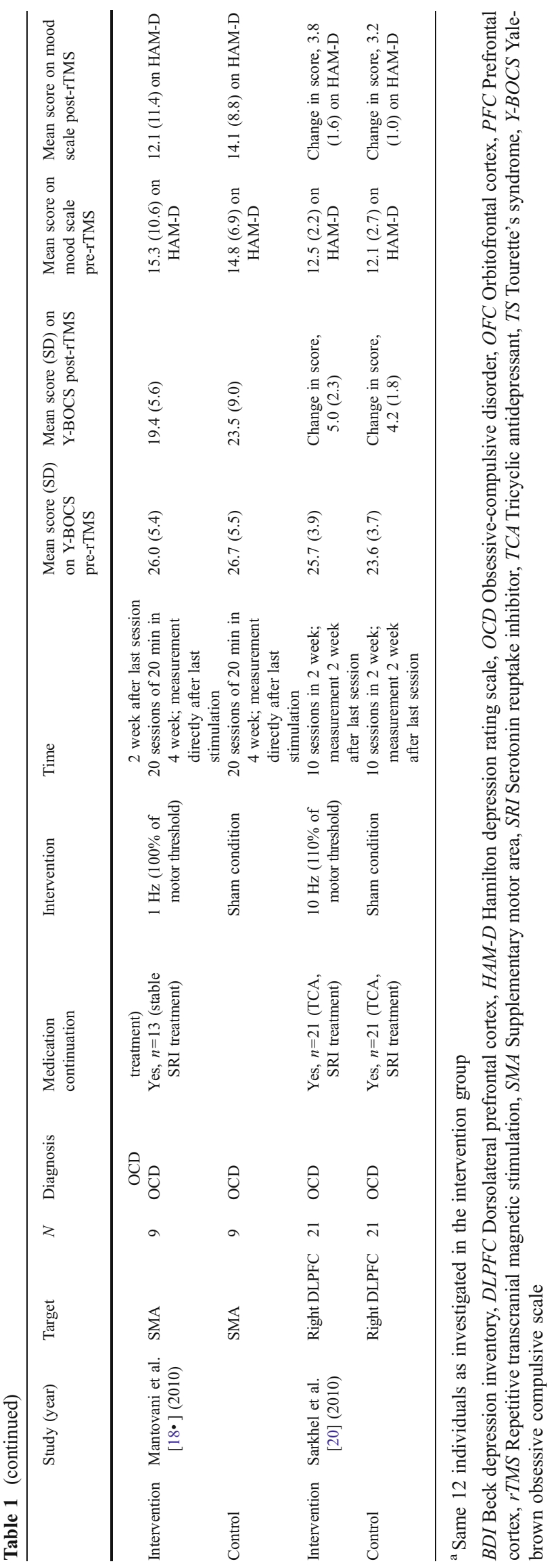

rTMS ( $1 \mathrm{~Hz}, 110 \%$ motor threshold) in addition to ongoing serotonin reuptake inhibitor treatment. After 2 weeks of follow-up, obsessive-compulsive symptoms improved, with mean Y-BOCS reductions of $28 \%$, but no differences between sham and real rTMS were observed. The authors concluded that rTMS did not result in an effect on OCD by stimulating the left DLPFC. Similarly, in a study by Sachdev et al. [12], high-frequency $(10 \mathrm{~Hz}, 110 \%$ motor threshold) rTMS of the left DLPFC yielded significant YBOCS decreases (6.0 points, 23.1\%) over 2 weeks in 10 OCD patients, but the effects were similar after sham rTMS in 8 patients (5.0 points, $20.4 \%$ ).

Finally, in an Indian sham-controlled study, active rTMS at $10 \mathrm{~Hz}, 110 \%$ motor threshold $(n=21)$ and sham rTMS $(n=$ 21 ) of the right DLPFC elicited similar improvement in obsessions and compulsions 2 weeks after the 10th session [20]. The Y-BOCS reduction for active rTMS was 5.0 points (19.5\%), and 4.2 points (17.7\%) for sham rTMS. Interestingly, depressive scores, as measured by the HAM-D, were reduced significantly over time in the real rTMS group compared with the sham rTMS group $(P>0.04)$. A total of $76.2 \%$ of those receiving real rTMS were partial responders (25\% reduction in HAM-D scores from baseline), compared with $66.7 \%$ in the sham group. The authors concluded that right DLPFC rTMS has no effect on OCD but is modestly effective in the treatment of comorbid depressive symptoms.

In conclusion, in open-label studies, high-frequency rTMS of the right and/or left DLPFC appears to be effective in reducing obsessive-compulsive symptoms. However, this could not be replicated in double-blind, sham-controlled studies. In those studies, neither low nor high rTMS and neither rTMS to the left nor to the right DLPFC appeared to be more effective than sham rTMS.

\section{Orbitofrontal Cortex}

In 2009, Ruffini and colleagues [13] examined the OFC as a new target for rTMS in drug-resistant OCD patients. The participants received $10 \mathrm{~min}$ of $1-\mathrm{Hz}$ rTMS at $80 \%$ motor threshold for 15 sessions to the left OFC; however, the coil was placed parallel (active, $n=16$ ) or perpendicular (sham, $n=7)$ to the scalp. They found significant reduction of YBOCS scores comparing active versus sham treatment for 10 weeks after the end of rTMS $(P<0.02)$, with loss of significance after 12 weeks $(P<0.06)$. Y-BOCS reduction was $19.7 \%$ immediately after rTMS and $14.7 \%$ after 12 weeks of follow-up, but only $6.7 \%$ and $5.7 \%$, respectively, for the sham condition. There was also a benefit in terms of depressive and anxiety symptoms, but not at a significant level in the two groups. Similar to findings for DLPFC rTMS, this study suggests that lowfrequency rTMS of the OFC may only acutely improve obsessive-compulsive symptoms. 
Supplementary Motor Area

Two groups investigated the efficacy of low rTMS to the SMA in addition to ongoing pharmacotherapy. In 2006, Mantovani et al. [14] conducted an open-label study of 10 patients with OCD, Tourette's syndrome, or both. Individuals were treated with active rTMS to the SMA for 10 daily sessions at $1 \mathrm{~Hz}, 100 \%$ motor threshold. After 2 weeks of daily rTMS, the Y-BOCS reduction (28.6\%) and HAM-D reduction $(47.8 \%)$ were both significant, and they remained stable after 3 months' follow-up in the OCD as well as in the OCD/Tourette's syndrome group. In 2010, the same group examined rTMS (at $1 \mathrm{~Hz}$ and $100 \%$ motor threshold) to the SMA bilaterally in a randomized, sham-controlled, double-blind design [18]. After 4 weeks of stimulation, the Y-BOCS decreased significantly $(P<0.001)$ in the active group (6 points, $25.4 \%$ ) and the sham group (3.2 points, $12.0 \%$ ) without significant differences between the two treatment conditions.

Finally, an open, sham-controlled study investigated the possible therapeutic effects and safety of sequentially combined low-frequency $(1 \mathrm{~Hz}, 110 \%$ threshold) rTMS to the right DLPFC and the SMA in 10 patients with treatment-resistant OCD [17]. Similar improvements in obsessive-compulsive and depressive symptoms were observed for sham and real rTMS at 2 weeks after the last of 14 sessions. The Y-BOCS reductions were 2.9 points $(10.9 \%)$ and 3.4 points $(12.9 \%)$ for real and sham rTMS, respectively. rTMS was a safe method, and there was no significant change in cognitive functioning after stimulation. Similar to DLPFC and OFC stimulation, rTMS to the SMA was a safe method to immediately improve obsessivecompulsive symptoms; however, improvement did not linger on over time.

In conclusion, efficacy of low- and high-frequency rTMS to the left or right DLPFC, the OFC, or the SMA has been investigated in a total of 110 obsessivecompulsive patients over the past decade. Although open studies have initially demonstrated beneficial effects of rTMS on obsessive-compulsive and depressive symptoms during the first hours after stimulation, these effects disappeared during follow-up and, more importantly, rTMS did not show any advantages over sham stimulation in double-blind, sham-controlled studies.

\section{Side Effects and Safety}

rTMS is generally regarded as a safe and noninvasive therapeutic technique. Although extremely rare, the most severe acute adverse effect related to rTMS is the induction of epileptic seizures. The chance of getting a seizure during high-frequency rTMS is greater than during low-frequency
rTMS. Other side effects that have been reported are induction of hypomania, local pain, headache, paresthesia, hearing changes, and thyroid-stimulating hormone and blood lactate level changes. The two latter have only been reported in high rTMS [22].

In the studies of rTMS in OCD patients, low-frequency rTMS study patients occasionally reported headache or localized scalp pain $[14,16,17 \cdot]$, whereas in the highfrequency rTMS patients, side effects were more often noted. The most common complaint in those studies was headache, followed by localized scalp pain, facial nerve stimulation, fainting, and weepiness [12, 20, 21]. None of the side effects held on longer than 4 weeks after stimulation, and neither serious adverse events such as seizures and memory problems nor cognition problems were disclosed.

\section{Conclusions and Future Directions}

Since 1997, rTMS has been applied as an experimental treatment in cases of refractory OCD. Local induction of a depolarizing magnetic field pulse may decrease obsessivecompulsive symptoms by normalizing hypermetabolism in orbitofrontal-striatal circuits. The technique is noninvasive and yields no side effects or mild side effects, of which headache is the most common. Because of the lack of studies with comparable stimulation or treatment parameters and with reliable designs, it is difficult to draw clear conclusions; this corresponds with a Cochrane review from 2003 about TMS treatment in OCD [23]. Explorations of rTMS to the DLPFC, OFC, or SMA in a total of 10 studies have demonstrated only acute efficacy for obsessivecompulsive symptoms of rTMS and no differences with sham treatment.

To generalize the results of these studies, further research is necessary. Careful consideration of target regions and stimulation parameters, longer follow-up, and the use of a double-blind, sham-controlled design may allow us to draw founded conclusions in the future. Besides, as the efficacy of rTMS is often time limited, the necessity of a second rTMS after several weeks should be investigated. Moreover, functional MRI studies of rTMS in OCD are needed to clarify the specific stimulation region of rTMS. Nevertheless, rTMS may play an important role in research settings. For example, rTMS could be used to modulate obsessive-compulsive symptoms and brain activity in functional MRI and receptor-binding studies. Otherwise, as the improvement of symptoms is often noted in sham settings, it would be interesting to investigate the neural underpinnings of the placebo effect caused by sham rTMS. Finally, a novel stimulation paradigm was recently designed: theta-burst stimulation, a low-intensity burst of 
rTMS at $50 \mathrm{~Hz}$ as a safer, more consistent, and longer lasting rTMS [24]. The results of the first case study with this paradigm in OCD and depression are promising and warrant further exploration [25•].

Disclosure No potential conflicts of interest relevant to this article were reported.

Open Access This article is distributed under the terms of the Creative Commons Attribution Noncommercial License which permits any noncommercial use, distribution, and reproduction in any medium, provided the original author(s) and source are credited.

\section{References}

Papers of particular interest, published recently, have been highlighted as:

- Of importance

1. Heyman I, Mataix-Cols D, Fineberg NA. Obsessive-compulsive disorder. BMJ. 2006;333:424-9.

2. Bruijn DC, Beun S, de GR, ten HM, Denys D. Subthreshold symptoms and obsessive-compulsive disorder: evaluating the diagnostic threshold. Psychol Med. 2010;40:989-97.

3. Fullana MA, Mataix-Cols D, Caspi A, Harrington H, Grisham JR, Moffitt TE, et al. Obsessions and compulsions in the community: prevalence, interference, help-seeking, developmental stability, and co-occurring psychiatric conditions. Am J Psychiatry. 2009;166:329-36.

4. Denys D. Pharmacotherapy of obsessive-compulsive disorder and obsessive-compulsive spectrum disorders. Psychiatr Clin North Am. 2006;29:553-84. xi.

5. Barker AT, Jalinous R, Freeston IL. Non-invasive magnetic stimulation of human motor cortex. Lancet. 1985;1:1106-7.

6. Whiteside SP, Port JD, Abramowitz JS. A meta-analysis of functional neuroimaging in obsessive-compulsive disorder. Psychiatry Res. 2004;132:69-79.

7. Greenberg BD, George MS, Martin JD, Benjamin J, Schlaepfer TE, Altemus M, et al. Effect of prefrontal repetitive transcranial magnetic stimulation in obsessive-compulsive disorder: a preliminary study. Am J Psychiatry. 1997;154:867-9.

8. George MS, Wassermann EM, Williams WA, Steppel J, PascualLeone A, Basser P, et al. Changes in mood and hormone levels after rapid-rate transcranial magnetic stimulation (rTMS) of the prefrontal cortex. J Neuropsychiatry Clin Neurosci. 1996;8:172-80.

9. Siebner HR, Rothwell J. Transcranial magnetic stimulation: new insights into representational cortical plasticity. Exp Brain Res. 2003;148:1-16.

10. Speer AM, Kimbrell TA, Wassermann EM, Repella D, Willis MW, Herscovitch P, et al. Opposite effects of high and low frequency rTMS on regional brain activity in depressed patients. Biol Psychiatry. 2000;48:1133-41.

11. Baxter Jr LR, Phelps ME, Mazziotta JC, Guze BH, Schwartz JM, Selin CE. Local cerebral glucose metabolic rates in obsessive- compulsive disorder. A comparison with rates in unipolar depression and in normal controls. Arch Gen Psychiatry. 1987;44:211-8.

12. Sachdev PS, Loo CK, Mitchell PB, McFarquhar TF, Malhi GS. Repetitive transcranial magnetic stimulation for the treatment of obsessive compulsive disorder: a double-blind controlled investigation. Psychol Med. 2007;37:1645-9.

13. Ruffini C, Locatelli M, Lucca A, Benedetti F, Insacco C, Smeraldi E. Augmentation effect of repetitive transcranial magnetic stimulation over the orbitofrontal cortex in drug-resistant obsessive-compulsive disorder patients: a controlled investigation. Prim Care Companion J Clin Psychiatry. 2009;11:226-30.

14. Mantovani A, Lisanby SH, Pieraccini F, Ulivelli M, Castrogiovanni $\mathrm{P}$, Rossi S. Repetitive transcranial magnetic stimulation (rTMS) in the treatment of obsessive-compulsive disorder (OCD) and Tourette's syndrome (TS). Int J Neuropsychopharmacol. 2006;9:95-100.

15. Picard N, Strick PL. Imaging the premotor areas. Curr Opin Neurobiol. 2001;11:663-72.

16. Alonso P, Pujol J, Cardoner N, Benlloch L, Deus J, Menchon JM, et al. Right prefrontal repetitive transcranial magnetic stimulation in obsessive-compulsive disorder: a double-blind, placebocontrolled study. Am J Psychiatry. 2001;158:1143-5.

17. • Kang JI, Kim CH, Namkoong K, Lee CI, Kim SJ. A randomized controlled study of sequentially applied repetitive transcranial magnetic stimulation in obsessive-compulsive disorder. J Clin Psychiatry. 2009;70:1645-51. This study investigated the efficacy of rTMS in OCD in a double-blind, randomized, sham-controlled design.

18. - Mantovani A, Simpson HB, Fallon BA, Rossi S, Lisanby SH. Randomized sham-controlled trial of repetitive transcranial magnetic stimulation in treatment-resistant obsessive-compulsive disorder. Int J Neuropsychopharmacol. 2010;13:217-27. This study also investigated the efficacy of rTMS in OCD in a double-blind, randomized, sham-controlled design.

19. Prasko J, Paskova B, Zalesky R, Novak T, Kopecek M, Bares M, et al. The effect of repetitive transcranial magnetic stimulation (rTMS) on symptoms in obsessive compulsive disorder. A randomized, double blind, sham controlled study. Neuro Endocrinol Lett. 2006;27:327-32.

20. Sarkhel S, Sinha VK, Praharaj SK. Adjunctive high-frequency right prefrontal repetitive transcranial magnetic stimulation (rTMS) was not effective in obsessive-compulsive disorder but improved secondary depression. J Anxiety Disord. 2010;24:535-9.

21. Sachdev PS, McBride R, Loo CK, Mitchell PB, Malhi GS, Croker VM. Right versus left prefrontal transcranial magnetic stimulation for obsessive-compulsive disorder: a preliminary investigation. J Clin Psychiatry. 2001;62:981-4.

22. Rossi S, Hallett M, Rossini PM, Pascual-Leone A. Safety, ethical considerations, and application guidelines for the use of transcranial magnetic stimulation in clinical practice and research. Clin Neurophysiol. 2009;120:2008-39.

23. Martin JL, Barbanoj MJ, Perez V, Sacristan M. Transcranial magnetic stimulation for the treatment of obsessive-compulsive disorder. Cochrane Database Syst Rev 2003;CD003387.

24. Huang YZ, Edwards MJ, Rounis E, Bhatia KP, Rothwell JC. Theta burst stimulation of the human motor cortex. Neuron. 2005;45:201-6.

25. • Wu CC, Tsai CH, Lu MK, Chen CM, Shen WC, Su KP. Thetaburst repetitive transcranial magnetic stimulation for treatmentresistant obsessive-compulsive disorder with concomitant depression. J Clin Psychiatry. 2010;71:504-6. This article describes in a case study a promising novel stimulation paradigm for OCD: theta-burst stimulation. 